\title{
Goal-driven Structured Argumentation for Patient Management in a Multimorbidity Setting
}

\author{
Tiago Oliveira ${ }^{1}$, Jérémie Dauphin ${ }^{2}$, Ken $\mathrm{Satoh}^{3}$, Shusaku Tsumoto ${ }^{4}$, and Paulo \\ Novais $^{5}$ \\ 1 MESM, Tokyo Medical and Dental University, Tokyo, Japan \\ tiago.oliveira.mesm@mri.tmd.ac.jp \\ 2 CSC, University of Luxembourg, Esch-sur-Alzette, Luxembourg \\ jeremie.dauphin@uni.lu \\ 3 National Institute of Informatics, Tokyo, Japan \\ ksatoh@nii.co.jp \\ 4 Shimane University, Izumo, Japan \\ tsumoto@med.shimane-u.ac.jp \\ 5 Algoritmi Centre, University of Minho, Braga, Portugal \\ pjon@di.uminho.pt
}

\begin{abstract}
We use computational argumentation to both analyse and generate solutions for reasoning in multimorbidity about consistent recommendations, according to different patient-centric goals. Reasoning in this setting carries a complexity related to the multiple variables involved. These variables reflect the co-existing health conditions that should be considered when defining a proper therapy. However, current Clinical Decision Support Systems (CDSSs) are not equipped to deal with such a situation. They do not go beyond the straightforward application of the rules that build their knowledge base and simple interpretation of Computer-Interpretable Guidelines (CIGs). We provide a computational argumentation system equipped with goal-seeking mechanisms to combine independently generated recommendations, with the ability to resolve conflicts and generate explanations for its results. We also discuss its advantages over and relation to Multiple-criteria Decisionmaking (MCDM) in this particular setting.
\end{abstract}

\section{Introduction}

Multimorbidity is the presence of two or more chronic medical conditions in an individual. It is a complex situation, particularly when the number of existing conditions is high and there are treatment conflicts [25. These conflicts are typically: drug-drug interactions, when treatments have a negative combined effect on the patient; and drug-disease interactions, when a treatment for a condition negatively affects the evolution of another condition. Clinical Decision Support Systems (CDSSs) based on Computer-Intepretable Guidelines (CIGs) are not 
capable of combining different CIG executions to address multiple health conditions, as CIGs are designed to handle a single disease 2010. Furthermore, computational approaches that aim to tackle this problem [26]27] are limited in the dimensions of multimorbidity they consider, namely when it comes to: patient preferences, patient-specific prioritized goals, and decidable mechanisms for conflict resolution. These dimensions are considered to be fundamental in reasoning for patient management and, in what preferences and goals are concerned, should result from a discussion between patient and health care professional [15|24. In recent years, several works call for the use of Multiple Criteria Decision-making (MCDM) methods to address conflicts in medical decision-making, produce decisions based on priorities over various criteria, and handle complexity in this setting [312. However, a general MCDM method lacks the ability to explain and justify decisions and lay out their respective implications. These elements become opaque when scores are computed and presented.

We explore structured argumentation to formalize conflict resolution in multimorbidity and compute aggregated consistent sets of CIG recommendations that take into account the above-mentioned dimensions. The appeal of argumentation in comparison to other computational approaches is in reasoning with conflicting and incomplete information in a way that aims to emulate human reasoning, while allowing important conflicts to be highlighted and analyzed [2]. We augment the ASPIC+ [13] argumentation system for it provides sub-argument structure, important for explanations, and has been extensively studied and justified in regards to semantics for preferences over defeasible rules and mechanisms to perform defeasible reasoning. It also allows for contraposition, a useful feature when analyzing conflicts. We propose the ASPIC $+\mathrm{G}$ argumentation system for multimorbidity as a goal-driven argumentation system to select best solutions and map basic elements in multimorbidity reasoning to it. Following from this, we also provide a reasoning framework that takes into account drug-drug and drug-disease conflicts, patient preferences, and prioritized patient-specific goals. We demonstrate that the selected best solution can be used to derive in-depth explanations and provide mechanisms to produce justifications for conclusions. Finally, we show that the proposed argumentation system subsumes MCDM, with the added benefit of providing explanations for multimorbidity decisions.

\section{Case Example}

The case example to demonstrate the computational argumentation framework was adapted from a clinical case in [22, simplified for the sake of brevity and understanding. There are slight differences such as the addition of chronic kidney disease to showcase reasoning features.

Example 1. Patient A has a history of type 2 diabetes. Upon consultation and the completion of medical exams, it was possible to conclude that the patient, besides type 2 diabetes, has obesity, hypertension, and chronic kidney disease. The case is run in a CDSS with CIG agents that handle each health condition separately, yielding recommendations: 
- CIG Agent 1 (for obesity): Define weight decrease $(w d)$ as a therapy goal. To reduce weight, the patient should practice diet and exercise (de) [18.

- CIG Agent 2 (for diabetes): Define blood glucose decrease $(g d)$ as a therapy goal. Sulfonylurea (sulf) or meglitinide ( $m e g)$ can reduce blood glucose elevations, but they cause weight increase (wi). Metformin (met) can lower blood glucose, but its use in the presence of chronic kidney disease $(c k d)$ should be avoided as it may accelerate chronic kidney disease $(a c k d)$. The patient should only take one of the drugs 19 .

- CIG Agent 3 (for kidney disease): Define delay chronic kidney disease $(d c k d)$ as a therapy goal. The patient is advised to take angiotensin converting enzyme inhibitors (acei) as they delay the progression of chronic kidney disease to kidney failure [16].

- CIG Agent 4 (for hypertension): Define blood pressure decrease (bpd) as a therapy goal. Administer an angiotensin converting enzyme inhibitor (acei) or a calcium channel blocker $(c c b)$ to decrease blood pressure. However, a calcium channel blocker compromises the effectiveness of glucose control drugs such as meglitinide or metformin [17.

Following the four CIG agents separately would produce drug-disease interactions. When considering CIG Agent 1 and CIG Agent 2, there is a conflict with the use of sulfonylurea and meglitinide from CIG Agent 2, as these drugs cause weight increase and this effect is contrary to the therapy goal outlined for obesity in CIG Agent 1, weight decrease. When adding the recommendations of CIG Agent 3 to the first two, other conflicts appear. The use of metformin for the treatment of diabetes is compromised by the recommendation to avoid the use of metformin in the presence of chronic kidney disease. Additionally, from CIG Agent 4, the recommendation to take a calcium channel blocker compromises the effectiveness of metformin and meglitinide. This is a drug-drug interaction. The mutually exclusive use of drugs for diabetes also constitutes drug-drug interactions.

In practice, the case is handled by establishing a priority over patient-specific goals and eliciting patient preferences [15. Reproducing the source of the example 22, the health care professional acknowledges obesity is the most severe issue for the patient, thus weight decrease is the most preferred goal, followed by blood glucose decrease as the second goal, with blood pressure decrease at the same level. Delaying kidney disease is the least preferred goal. Additionally, the patient shows a clear preference for sulfonylurea or meglitinide, as the use of metformin has caused him severe adverse reactions in the past.

The knowledge enclosed in CIGs typically follows a task network model where each element is a task to carry out. A task recommending a treatment is normally called an Action and contains certain structured information about the treatments to be applied, respective outcomes and pre-conditions for application reflecting interactions [20]. This is the task we focus on and the basis for CIG Agent recommendations. Further ahead we use Example 1 to instantiate AS$\mathrm{PIC}+\mathrm{G}$. The process of preference elicitation and goal prioritization, including 
the functions that bring them about (e.g. severity of disease), are outside the scope of this work, so they will only be referred to as examples.

\section{The ASPIC+G Argumentation System}

The intuition behind ASPIC+G is that argumentation is often driven by goals which reflect the multiple objectives that may be achieved in a discussion. This fits reasoning in a multimorbidity setting particularly well due to the goaloriented nature of the process.

\subsection{Definition and Argument Construction}

We define an ASPIC $+\mathrm{G}$ argumentation theory as follows.

Definition 1. An argumentation theory in $A S P I C+G$ is a tuple $\left\langle\mathcal{L}, \mathcal{R}, \mathrm{n}, \leqslant_{\mathcal{R}_{d}}, \mathcal{G}, \leqslant \mathcal{G}\right\rangle$, where:

$-\mathcal{L}$ is a logical language closed under negation $(\neg)$.

$-\mathcal{R}=\mathcal{R}_{s} \cup \mathcal{R}_{d}$ is a set of strict $\left(\mathcal{R}_{s}\right)$ and defeasible $\left(\mathcal{R}_{d}\right)$ rules of the form $\phi_{1}, \ldots, \phi_{n} \rightarrow \phi$ and $\phi_{1}, \ldots, \phi_{n} \Rightarrow \phi$ respectively, where $n \geq 0$ and $\phi_{i}, \phi \in \mathcal{L}$;

$-n$ is a partial function s.t. ${ }^{6} n: \mathcal{R} \rightarrow \mathcal{L}$;

$-\leqslant \mathcal{R}_{d}$ is a partial pre-order over defeasible rules $\mathcal{R}_{d}$, denoting a preference relation, with a strict counterpart $<_{\mathcal{R}_{d}}$ given by $X<_{\mathcal{R}_{d}} Y$ iff $X \leqslant_{\mathcal{R}_{d}} Y$ and $Y \nless_{\mathcal{R}_{d}} X$;

$-\mathcal{G} \subseteq \mathcal{L}$ is a set of goals that the arguments will try to fulfil s.t. $\forall \theta \in \mathcal{G}$, there exists a rule $\phi_{1}, \ldots, \phi_{n} \rightarrow \phi$ in $\mathcal{R}_{s}$ or $\phi_{1}, \ldots, \phi_{n} \Rightarrow \phi$ in $\mathcal{R}_{d}$ s.t. $\phi=\theta$;

- $\leqslant_{\mathcal{G}}$ is a total pre-order on $\mathcal{G}$, denoting preferences over goals, with $<_{\mathcal{G}}$ given by $\phi<_{\mathcal{G}} \psi$ iff $\phi \leqslant_{\mathcal{G}} \psi$ and $\psi \nless_{\mathcal{G}} \phi$, and $\simeq_{\mathcal{G}}$ given by $\phi \simeq_{\mathcal{G}} \psi$ iff $\phi \leqslant_{\mathcal{G}} \psi$ and $\psi \leqslant \mathcal{G} \phi$.

In $\mathrm{ASPIC}+\mathrm{G}$, knowledge is represented either as strict rules or defeasible rules. Therefore, an undisputable fact is a strict rule with empty antecedents and a disputable fact is represented as a defeasible rule with empty antecedents. The relation $\leqslant_{\mathcal{G}}$ is a total pre-order which allows for equally preferred goals, as it is often the case that distinctions between goals cannot be specified. In order to understand the construction of arguments, we specify functions to convey argument features. Conc(A) denotes the conclusion of argument A. Sub(A) denotes the set of sub-arguments of A. DefRules(A) denotes the set of all defeasible rules used in A. Finally, TopRule(A) denotes the last inference rule used in the argument. We use the following definition for argument construction, adapted from [13.

Definition 2. An argument $\mathrm{A}$ of an argumentation theory $\left\langle\mathcal{L}, \mathcal{R}, \mathrm{n}, \leqslant_{\mathcal{R}_{d}}, \mathcal{G}, \leqslant_{\mathcal{G}}\right\rangle$ has one of the following forms:

\footnotetext{
${ }^{6}$ s.t.: such that
} 


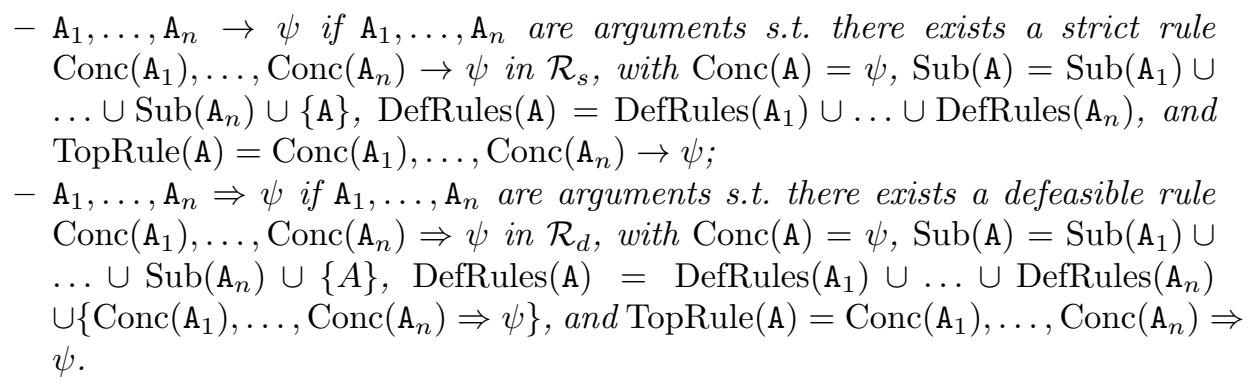

\subsection{Attack, Defeat, and Goal Fulfilment}

Attacks follow two of the three possible ways in ASPIC+ [13]. Arguments may be attacked on a conclusion of a defeasible inference (rebutting) or on a defeasible inference step itself (undercutting). Undermining attacks are represented as a special case of rebuttal. It is considered that an argument cannot be attacked on the conclusion of a strict inference. To define an undercutting attack, $\mathrm{n}$ is used to assign elements of $\mathcal{R}_{d}$ a well-formed formula in $\mathcal{L}$. $\mathrm{n}(r): r \in \mathcal{R}_{d}$ denotes that $r$ is applicable and $\neg \mathrm{n}(r)$ denotes that $r$ is not applicable. An argument using $r$ is undercut by any argument concluding $\neg \mathrm{n}(r)$. The following definition of attack was adapted from [13.

Definition 3. An argument $\mathrm{A}$ attacks an argument $\mathrm{B}$ iff $\mathrm{A}$ undercuts or rebuts $\mathrm{B}$, where: $\mathrm{A}$ undercuts $\mathrm{B}$ (on $\left.\mathrm{B}^{\prime}\right)$ iff $\operatorname{Conc}(\mathrm{A})=\neg n(r)$ for some $\mathrm{B}^{\prime} \in \operatorname{Sub}(\mathrm{B})$ s.t. the top rule $r$ of $\mathrm{B}^{\prime}$ is defeasible; $\mathrm{A}$ rebuts $\mathrm{B}$ (on $\mathrm{B}^{\prime}$ ) iff $\operatorname{Conc}(\mathrm{A})=\neg \phi$ for some $\mathrm{B}^{\prime} \in \operatorname{Sub}(\mathrm{B})$ of the form $\mathrm{B}_{1}^{\prime \prime}, \ldots, \mathrm{B}_{n}^{\prime \prime} \Rightarrow \phi$.

The introduction of goals in argumentation demands the definition of a fulfilment relation.

Definition 4. An argument A fulfils goal $\theta \quad \in \quad \mathcal{G}$ iff Conc(A) $=\theta$. If A fulfils a goal, we denote it with Goal(A). For a set of arguments $S$, we write $\operatorname{Goal}(S)$ for the set of goals fulfilled by the arguments in $S$, i.e. $\operatorname{Goal}(S)=\{\operatorname{Goal}(\mathrm{A}) \mid \mathrm{A} \in S$ s.t. A fulfils a goal $\}$.

We use a preference order over arguments $\preceq$ determined by a weakest-link principle on $\leqslant_{\mathcal{R}_{d}}$, as described in [13. To specify $\preceq$, we resort to an ordering of defeasible rule sets $\unlhd_{\mathcal{R}_{d}}$, defined over an elitist criterion, i.e., the set with the overall weakest rule is the weakest. Therefore, given two sets of defeasible rules $R$ and $R^{\prime}$ : if $R=\emptyset$ then $R \unlhd_{\mathcal{R}_{d}} R^{\prime}$; if $R=\emptyset$ and $R^{\prime} \neq \emptyset$ then $R^{\prime} \unlhd_{\mathcal{R}_{d}} R$; else, assuming a pre-order $\leqslant \mathcal{R}_{d}$ over the elements in $R \cup R^{\prime}$, if $\exists X \in R$ s.t. $\forall Y \in R^{\prime}$, $X \leqslant_{\mathcal{R}_{d}} Y$, then $R \unlhd_{\mathcal{R}_{d}} R^{\prime}$.

Considering two arguments A and B, we say that A $\preceq$ B iff DefRules(A) $\unlhd_{\mathcal{R}_{d}}$ $\operatorname{DefRules}(\mathrm{B})$. We can define the strict counterpart $\prec$ directly under the weakestlink principle, in terms of $\unlhd_{\mathcal{R}_{d}}$.

Attack and argument preference bring about a defeat relation $\mathcal{D}$. It is considered that: an argument A successfully rebuts an argument B if A rebuts B on B' 
and $\mathrm{A} \nprec \mathrm{B}^{\prime}$; an argument $\mathrm{A}$ defeats an argument $\mathrm{B}$ iff $\mathrm{A}$ undercuts or successfully rebuts $B$.

We now define an ASPIC $+\mathrm{G}$ framework as follows.

Definition 5. An argumentation framework in $A S P I C+G$ is a tuple $\left(\mathcal{A}, \mathcal{D}, \mathcal{G}, \leqslant_{\mathcal{G}}, \mathcal{F}\right)$, where $\mathcal{A}$ is a set of arguments, $\mathcal{D} \subseteq \mathcal{A} \times \mathcal{A}$ is a binary relation of defeat, $\mathcal{G}$ is the set of goals, $\leqslant_{\mathcal{G}}$ is a preference order over goals, and $\mathcal{F}$ is a binary relation of fulfilment s.t. $\mathcal{F} \subseteq \mathcal{A} \times \mathcal{G}$.

To select arguments, the framework uses the semantics presented in Dung's abstract argumentation framework [7, according to the following definition.

Definition 6. Let $\left(\mathcal{A}, \mathcal{D}, \mathcal{G}, \leqslant_{\mathcal{G}}, \mathcal{F}\right)$ be an $A S P I C+G$ argumentation framework. For any $\mathrm{X} \in \mathcal{A}, \mathrm{X}$ is acceptable with respect to some set $S \subseteq \mathcal{A}$ iff $\forall \mathrm{Y} \in \mathrm{A}$ s. t. $(\mathrm{Y}, \mathrm{X}) \in \mathcal{D}, \exists \mathrm{Z} \in S$ s.t. $(\mathrm{Z}, \mathrm{Y}) \in \mathcal{D}$. Let $S \subseteq \mathcal{A}$ be a conflict free set, i.e., there are no $\mathrm{A}, \mathrm{B}$ in $S$ s.t. $(\mathrm{A}, \mathrm{B}) \in \mathcal{D}$. Then: $S$ is an admissible extension iff $\mathrm{X} \in S$ implies $\mathrm{X}$ is acceptable with respect to $S$; and $S$ is a preferred extension iff it is a set inclusion maximal admissible extension.

It is of interest within the context of multimorbidity to produce the preferred, and thus maximal sets of arguments, which are the most inclusive self-defended sets, containing all the sub-arguments that lead to a conclusion and the arguments that defend it.

\subsection{Goal Set Ordering}

The preferred extensions are viewed as consistent argumentation paths in the discussion and the possible solutions to solve a problem. However, it is necessary to compare the sets of goals that they fulfil in order to rank them. We now define a goal set ordering $\unlhd_{\mathcal{G}}$ over sets of goals.

Definition 7. Let $S$ and $S^{\prime}$ be two finite sets of goals. We define the goal set ordering, denoted by the operator $\unlhd_{\mathcal{G}}$, as: $S^{\prime} \unlhd_{\mathcal{G}} S$ iff $S^{\prime}=\emptyset$ or $\exists g \in\left(S \backslash S^{\prime}\right)$ such that $\forall g^{\prime} \in\left(S^{\prime} \backslash S\right), g^{\prime} \leqslant_{\mathcal{G}} g$.

A goal set ordering $S^{\prime} \unlhd_{\mathcal{G}} S$ denotes that $S$ is at least as preferred as $S^{\prime}$, possibly more. The underlying principle is that the argumentation will always try to fulfil the goals by their order of importance. Due to the base relation $\leqslant \mathcal{G}$ being a total pre-order, $\unlhd_{\mathcal{G}}$ is also a total pre-order. We also allow for different goal extensions to be equally preferred by fulfilling goals of equal preference.

With the goal set ordering, it becomes possible to find the best goal-driven solutions, i.e., the top preferred extensions.

Definition 8. Let $F=\left(\mathcal{A}, \mathcal{D}, \mathcal{G}, \leqslant_{\mathcal{G}}, \mathcal{F}\right)$ be an $A S P I C+G$ argumentation framework and $S$ a preferred extension of $F$. We say that $S$ is a top preferred extension of F iff for every preferred extension $S^{\prime}$ of $F, \operatorname{Goal}\left(S^{\prime}\right) \unlhd_{\mathcal{G}} \operatorname{Goal}(S)$.

ASPIC $+\mathrm{G}$ will be used to model Example 1 and demonstrate the outcomes of reasoning in multimorbidity using patient preferences and patient-specific goals. 


\section{Modelling Multimorbidity with ASPIC+G}

We perform a mapping of basic components in CIG multimorbidity management to $\mathrm{ASPIC}+\mathrm{G}$ and demonstrate its reasoning features. We also demonstrate how the given solutions can produce explanations.

\subsection{Formalization and Reasoning}

Let us consider $\mathbb{A}$ as a set containing all aggregated Action tasks recommended by all CIG agents, such as the ones in Example 1. We denote an action $A_{x, a} \in \mathbb{A}$, where $x$ is the index and a the CIG agent recommending the action. For instance, $A_{1,2}$ is the first action recommended by CIG Agent 2. An action $\mathrm{A}_{\mathrm{x}, \mathrm{a}}$ is a tuple $\left\langle t_{x, a}, O_{x, a}, P_{x, a}\right\rangle$, where:

$-t_{x, a}$ is a treatment;

- $\mathrm{O}_{\mathrm{x}, \mathrm{a}}=\left\{\left(e_{1}, \mathrm{C}_{1}, \lambda_{1}\right), \ldots,\left(e_{n}, \mathrm{C}_{\mathrm{n}}, \lambda_{n}\right): n>0\right\}$ stands for outcomes and is a set containing effects $\left(e_{i}, \mathrm{C}_{\mathrm{i}}, \lambda_{i}\right), i \in\{1, \ldots, n\}$ brought about by treatment $\mathrm{t}_{\mathrm{x}, \mathrm{a}}$, where: $e_{i}$ is a description of an effect; $C_{\mathbf{i}}=\left\{c_{1}, \ldots, c_{m}: m \geq 0\right\}$ is a set with patient-specific conditions unifiable with the patient state $c_{j}, j \in\{1, \ldots, m\}$ that enable the occurrence of effect $e_{i}$ over treatment $t_{x, a} ; \lambda_{i}$ is the impact of an effect $e_{i}$, if $e_{i}$ is a positive effect, then $\lambda_{i}=\oplus$, otherwise, if it is a negative effect, $\lambda_{i}=\ominus$.

- $\mathrm{P}_{\mathrm{x}, \mathrm{a}}=\left\{p_{1}, \ldots, p_{n}: n \geq 0\right\}$ denotes pre-conditions and contains constraints for the application of a treatment $t_{x, a}$.

From Example 1, we have the following actions in $\mathbb{A}$ :

$\mathrm{A}_{1,1}\langle d e,\{(w d, \emptyset, \oplus)\}, \emptyset\rangle$;

$\mathrm{A}_{1,2}\langle\operatorname{sulf},\{(g d, \emptyset, \oplus),(w i, \emptyset, \ominus)\},\{\neg m e g, \neg m e t\}\rangle ;$

$\mathrm{A}_{2,2}\langle$ meg $,\{(g d, \emptyset, \oplus),(w i, \emptyset, \ominus)\},\{\neg s u l f, \neg m e t\}\rangle ;$

$\mathrm{A}_{3,2}\langle$ met $,\{(g d, \emptyset, \oplus),($ ackd, $\{$ ckd $\}, \ominus)\},\{\neg s u l f, \neg m e g\}\rangle ;$

$\mathrm{A}_{1,3}\langle$ acei, $\{(d c k d, \emptyset, \oplus)\}, \emptyset\rangle$;

$\mathrm{A}_{1,4}\langle$ acei $,\{(b p d, \emptyset, \oplus),(\neg(m e g \rightarrow g d), \emptyset, \ominus)\},\{\neg c c b\}\rangle ;$

$\mathrm{A}_{2,4}\langle c c b,\{(b p d, \emptyset, \oplus),(\neg(m e g \rightarrow g d), \emptyset, \ominus)\},\{\neg a c e i\}\rangle$.

While there are CIG languages, such as PROForma [6], that encode the impact $\lambda$ of an effect, this is not always the case. As such, we assume that this evaluation of effects is provided by either the CIG language or a joint assessment by health care professional and patient.

The next component of multimorbidity management is a set containing the contraries of effects $\mathbb{E}=\left\{C_{1}, \ldots, C_{n}: n \geq 0\right\}$ where each $C_{i}, i \in\{1, \ldots, n\}$, is a tuple $\left(e_{j}, e_{k}\right)$ s.t. $\exists \mathrm{A}_{\mathrm{x}, \mathrm{a}}=\left\langle\mathrm{t}_{\mathrm{x}, \mathrm{a}}, \mathrm{O}_{\mathrm{x}, \mathrm{a}}, \mathrm{P}_{\mathrm{x}, \mathrm{a}}\right\rangle, \mathrm{A}_{\mathrm{y}, \mathrm{b}}=\left\langle\mathrm{t}_{\mathrm{y}, \mathrm{b}}, \mathrm{O}_{\mathrm{y}, \mathrm{b}}, \mathrm{P}_{\mathrm{y}, \mathrm{b}}\right\rangle \in \mathbb{A}$, s.t. $\left(e_{j}, \mathrm{C}_{\mathrm{j}}, \lambda_{j}\right) \in \mathrm{O}_{\mathrm{x}, \mathrm{a}}$ and $\left(e_{k}, \mathrm{C}_{\mathrm{k}}, \lambda_{k}\right) \in \mathrm{O}_{\mathrm{y}, \mathrm{b}}$. Example 1 provides effect contraries: $\mathbb{E}=\{(w d, w i),(d c k d, a c k d)\}$. The automatic retrieval of contraries from a CIG language may be performed by analysing the clinical effects with medical terminologies and identifying the medical concept (e.g., weight) and term denoting a transition (e.g, increase, decrease), with a posterior matching with its opposite. Given the freedom associated with the expression of these effects, contrary identification is not addressed herein. 
The last component for reasoning in multimorbidity is the state of the patient $\mathbb{S}=\left\{s_{1}, \ldots, s_{n}: n \geq 0\right\}$, where each element is a condition manifested by the patient. In Example 1, we consider that $\mathbb{S}=\{c k d\}$, as this is the only element that interacts with the elements of other components.

With $\mathbb{A}, \mathbb{E}$ and $\mathbb{S}$ becomes possible to express applicability of treatments, treatment/effect relations, treatment conflicts, and effect conflicts in a logical language for ASPIC $+\mathrm{G}$. The purpose is to aggregate the knowledge elements provided by CIG agents through argumentation and augment them with patient preferences over treatments and treatment goals.

Definition 9. Let $\mathbb{A}, \mathbb{E}$ and $\mathbb{S}$, be the basic components for decision-making in multimorbidity. An argumentation theory in $A S P I C+G$ for multimorbidity is a tuple $\left\langle\mathcal{L}, \mathcal{R}, \mathrm{n}, \leqslant_{\mathcal{R}_{d}}, \mathcal{G}, \leqslant_{\mathcal{G}}\right\rangle$, where 7 .

- $\mathcal{R}=\mathcal{R}_{d} \cup \mathcal{R}_{s}$ are respectively defeasible and strict rules in which:

- $\mathcal{R}_{d}=\mathcal{R}_{1} \cup \mathcal{R}_{2}$ where $\mathcal{R}_{1}=\left\{\Rightarrow \mathrm{t}_{\mathrm{x}, \mathrm{a}} \mid \exists \mathrm{A}_{\mathrm{x}, \mathrm{a}}=\left\langle\mathrm{t}_{\mathrm{x}, \mathrm{a}}, \mathrm{O}_{\mathrm{x}, \mathrm{a}}, \mathrm{P}_{\mathrm{x}, \mathrm{a}}\right\rangle \in \mathbb{A}.\right\}$ and $\mathcal{R}_{2}=$ $\left\{\mathrm{t}_{\mathrm{x}, \mathrm{a}}, c_{1}, \ldots, c_{n} \Rightarrow e_{z} \mid \exists \mathrm{A}_{\mathrm{x}, \mathrm{a}}=\left\langle\mathrm{t}_{\mathrm{x}, \mathrm{a}}, \mathrm{O}_{\mathrm{x}, \mathrm{a}}, \mathrm{P}_{\mathrm{x}, \mathrm{a}}\right\rangle \in \mathbb{A},\left(e_{z},\left\{c_{1}, \ldots, c_{n}\right\}, \oplus\right) \in\right.$ $\left.\mathrm{O}_{\mathrm{x}, \mathrm{a}}, n \geq 0\right\}$

- $\mathcal{R}_{s}=\mathcal{R}_{3} \cup \mathcal{R}_{4} \cup \mathcal{R}_{5} \cup \mathcal{R}_{6}$ where $\mathcal{R}_{3}=\left\{\mathrm{t}_{\mathrm{x}, \mathrm{a}}, c_{1}, \ldots, c_{n} \rightarrow e_{z} \mid \exists \mathrm{A}_{\mathrm{x}, \mathrm{a}}=\right.$ $\left.\left\langle\mathrm{t}_{\mathrm{x}, \mathrm{a}}, \mathrm{O}_{\mathrm{x}, \mathrm{a}}, \mathrm{P}_{\mathrm{x}, \mathrm{a}}\right\rangle \in \mathbb{A},\left(e_{z},\left\{c_{1}, \ldots, c_{n}\right\}, \ominus\right) \in \mathrm{O}_{\mathrm{x}, \mathrm{a}}, n \geq 0\right\}, \mathcal{R}_{4}=\left\{\mathrm{t}_{\mathrm{x}, \mathrm{a}} \rightarrow\right.$ $\left.\neg \mathrm{t}_{\mathrm{y}, \mathrm{b}} \mid \exists \mathrm{A}_{\mathrm{x}, \mathrm{a}}=\left\langle\mathrm{t}_{\mathrm{x}, \mathrm{a}}, \mathrm{O}_{\mathrm{x}, \mathrm{a}}, \mathrm{P}_{\mathrm{x}, \mathrm{a}}\right\rangle, \mathrm{A}_{\mathrm{y}, \mathrm{b}}=\left\langle\mathrm{t}_{\mathrm{y}, \mathrm{b}}, \mathrm{O}_{\mathrm{y}, \mathrm{b}}, \mathrm{P}_{\mathrm{y}, \mathrm{b}}\right\rangle \in \mathbb{A}, \neg \mathrm{t}_{\mathrm{y}, \mathrm{b}} \in \mathrm{P}_{\mathrm{x}, \mathrm{a}}\right\}$, $\mathcal{R}_{5}=\left\{e_{j} \rightarrow \neg e_{k} \mid\left(e_{j}, e_{k}\right) \in \mathbb{E}\right.$ or $\left(e_{k}, e_{j}\right) \in \mathbb{E}$, and $\mathcal{R}_{6}=\{\rightarrow s \mid s \in \mathbb{S}\} ;$

$-\leqslant \mathcal{R}_{d}$ is a partial pre-order over defeasible rules $\mathcal{R}_{1}$, denoting a preference relation over treatments;

$-\mathcal{G}=\left\{e_{1}, \ldots, e_{n} \mid n \geq 0, \exists \mathrm{A}_{\mathrm{x}, \mathrm{a}}=\left\langle\mathrm{t}_{\mathrm{x}, \mathrm{a}}, \mathrm{O}_{\mathrm{x}, \mathrm{a}}, \mathrm{P}_{\mathrm{x}, \mathrm{a}}\right\rangle \in \mathbb{A},\left(e_{n}, C_{n}, \oplus\right) \in \mathrm{O}_{\mathrm{x}, \mathrm{a}}\right\}$ is a set of goals in terms of the positive effects of treatments;

$-\leqslant_{\mathcal{G}}$ is a total pre-order over treatment goals in $\mathcal{G}$.

Note that the treatments provided by CIG agents are handled as disputable facts and, thus, represented as defeasible rules with empty antecedents in $\mathcal{R}_{1}$. This stems from treatments being viewed as interventions that could be applied to the patient, but may not, given the context. Therefore, this element is defeasible. As for the treatment/effect relations, they are handled in two possible ways. In $\mathcal{R}_{2}$, this relation is depicted as a defeasible rule, when the effect of a treatment is positive. We consider that treatments only create a presumption in favour of their positive effect. However, when it comes to negative effects, we adopt a more conservative approach in $\mathcal{R}_{3}$, for negative effects are considered as something that compromises their corresponding positive effects, and, in a goaldriven search of solutions it is important to maximize the possibility of achieving the most preferred goals. For this reason, we represent a relationship between treatments and negative effects as strict rules. This also allows, by contraposition, to obtain the negation of treatments that compromise positive effects. $\mathcal{R}_{4}$ represents drug-drug conflicts extracted from pre-conditions of actions, indicating that two treatments must not be combined. Similarly, $\mathcal{R}_{5}$ presents effects that are contrary to each other. This allows for the derivation of drug-disease

\footnotetext{
${ }^{7}$ We omit $\mathcal{L}$ and $\mathrm{n}$, as they are implicit from the formalization.
} 
conflicts. Finally, $\mathcal{R}_{6}$ is used to describe patient state, consisting of undisputable facts. Accordingly, we apply strict rules with no antecedents.

We now instantiate $\mathrm{ASPIC}+\mathrm{G}$ for Example 1 by Definition 9 , $\mathcal{L}$ consists of all atoms defined for Example 1 and their negations. $n, \mathcal{R}, \leqslant_{\mathcal{R}_{d}}, \mathcal{G}$, and $\leqslant_{\mathcal{G}}$ are as follows:

$-\mathcal{R}_{d}=\{\Rightarrow$ de, $\Rightarrow$ sulf, $\Rightarrow$ meg, $\Rightarrow$ met, $\Rightarrow$ acei, $\Rightarrow$ ccb $\} \cup\{d e \Rightarrow$ $w d$, sulf $\Rightarrow g d, r_{1}:$ meg $\Rightarrow g d, r_{2}:$ met $\Rightarrow g d$, acei, ckd $\Rightarrow d c k d$, acei $\Rightarrow$ $b p d, c c b \Rightarrow b p d\}$

$-\mathcal{R}_{s}=\left\{\right.$ sulf $\rightarrow$ wi, meg $\rightarrow$ wi, met, ckd $\rightarrow$ ackd, ccb $\left.\rightarrow \neg r_{1}, c c b \rightarrow \neg r_{2}\right\} \cup$ $\{$ sulf $\rightarrow \neg$ meg, sulf $\rightarrow \neg$ met, meg $\rightarrow \neg$ met, acei $\rightarrow \neg c c b\} \cup\{w d \rightarrow$ $\neg$ wi, ackd $\rightarrow \neg d c k d\} \cup\{\rightarrow c k d\}$;

$-\mathcal{R}=\mathcal{R}_{d} \cup \mathcal{R}_{s}$

$-\leqslant \mathcal{R}_{d}:(\Rightarrow$ met $)<_{\mathcal{R}_{d}}(\Rightarrow$ sulf $),(\Rightarrow$ met $)<_{\mathcal{R}_{d}}(\Rightarrow m e g) ;$

$-\mathcal{G}=\{w d, g d, d c k d, b p d\}$

$-\leqslant_{\mathcal{G}}: d c k d<_{\mathcal{G}} g d \simeq_{\mathcal{G}}$ bpd $<_{\mathcal{G}} w d$.

In $\mathcal{R}, \mathrm{ASPIC}+\mathrm{G}$ allows for the representation of a situation in which a treatment negates defeasible rules $r_{1}$ and $r_{2}$, meaning that there is a medical circumstance in which these rules do not apply. In turn, these rules, due to their nature, are defeasible. The remaining defeasible rules reflect the possible treatments for diabetes, kidney disease, obesity, and hypertension. We need not be exhaustive in the listing of treatment conflicts in actions and treatment contraries, since the negation of the antecedent is obtained by contraposition. The relation $\leqslant \mathcal{R}_{d}$ reflects the treatment preference of the patient for sulf or meg over met. The goal set $\mathcal{G}$ contains the goals driving the treatment and their preference order is specified in $\leqslant_{\mathcal{G}}$. These goals are selected from positive effects in actions. $w d$ is the most preferred goal since obesity is the most significant concern of the patient. $b p d$ and $g d$ are equally preferred. Lastly, $d c k d$ is the least preferred.

By Definition 2, we build the arguments $\mathcal{A}$ for the argumentation framework along with representation of goals $\mathcal{G}$ :

$\mathcal{A}=\left\{\mathrm{A}_{1}: \Rightarrow\right.$ de, $\mathrm{A}_{2}: \mathrm{A}_{1} \Rightarrow w d, \mathrm{~A}_{2}^{\prime}: \mathrm{A}_{2} \rightarrow \neg w i, \mathrm{~A}_{2}^{\prime \prime}: \mathrm{A}_{2}^{\prime} \rightarrow \neg$ sulf, $\mathrm{A}_{2}^{\prime \prime \prime}: \mathrm{A}_{2}^{\prime} \rightarrow$ $\neg$ meg, $\mathrm{B}_{1}: \Rightarrow$ sulf, $\mathrm{B}_{2}: \mathrm{B}_{1} \Rightarrow g d, \mathrm{~B}_{2}^{\prime}: \mathrm{B}_{1} \rightarrow \neg$ met, $\mathrm{B}_{2}^{\prime \prime}: \mathrm{B}_{1} \rightarrow \neg$ meg, $\mathrm{B}_{2}^{\prime \prime \prime}: \mathrm{B}_{1} \rightarrow$ $w i, \mathrm{~B}_{2}^{\prime \prime \prime \prime}: \mathrm{B}_{2}^{\prime \prime \prime} \rightarrow \neg w d, C_{1}: \Rightarrow$ meg, $\mathrm{C}_{2}: \mathrm{C}_{1} \rightarrow g d, \mathrm{C}_{2}^{\prime}: \mathrm{C}_{1} \rightarrow \neg$ met, $\mathrm{C}_{2}^{\prime \prime}: \mathrm{C}_{1} \rightarrow$ $\neg$ sulf, $\mathrm{C}_{2}^{\prime \prime \prime}: \mathrm{C}_{1} \rightarrow w i, \mathrm{C}_{2}^{\prime \prime \prime \prime}: \mathrm{C}_{2}^{\prime \prime \prime} \rightarrow \neg w d, \mathrm{D}_{1}: \Rightarrow$ met, $\mathrm{D}_{2}: \mathrm{D}_{1} \rightarrow g d, \mathrm{D}_{2}^{\prime}: \mathrm{D}_{1} \rightarrow$ $\neg$ meg, $\mathrm{D}_{2}^{\prime \prime}: \mathrm{D}_{1} \rightarrow \neg$ sulf, $\mathrm{E}_{1}: \Rightarrow$ acei, $\mathrm{E}_{1}^{\prime}: \rightarrow$ ckd, $\mathrm{D}_{2}^{\prime \prime \prime}: \mathrm{D}_{1}, \mathrm{E}_{1}^{\prime} \rightarrow$ ackd, $\mathrm{D}_{3}: \mathrm{D}_{2}^{\prime \prime \prime} \rightarrow$ $\neg d c k d, E_{2}: E_{1}, E_{1}^{\prime} \Rightarrow d c k d, \mathrm{E}_{3}: \mathrm{E}_{2} \rightarrow \neg a c k d, \mathrm{E}_{4}: \mathrm{E}_{1}^{\prime}, \mathrm{E}_{3} \rightarrow \neg$ met, $\mathrm{E}_{5}: \mathrm{E}_{1} \Rightarrow$ $b p d, \mathrm{E}_{6}: \mathrm{E}_{1} \Rightarrow \neg c c b, \mathrm{~F}_{1}: \Rightarrow c c b, \mathrm{~F}_{2}: \mathrm{F}_{1} \Rightarrow b p d, \mathrm{~F}_{1}^{\prime}: \mathrm{F}_{1} \rightarrow \neg a c e i, \mathrm{~F}_{1}^{\prime \prime}: F_{1} \rightarrow$ $\left.\neg r_{1}, \mathrm{~F}_{1}^{\prime \prime \prime}: \mathrm{F}_{1} \rightarrow \neg r_{2}\right\}$ $\mathcal{G}=\left\{\mathrm{G}_{1}: w d, \mathrm{G}_{2}: g d, \mathrm{G}_{3}: d c k d, \mathrm{G}_{4}: b p d\right\}$.

By Definition 3 , we are able to derive the attack relations among arguments. Additionally, by Definition 4, we establish the fulfilment relations between arguments and goals. Attacks, fulfilments, and sub-argument relations are represented in the graph of Fig. 1. The explanatory power of a graph, describing an ASPIC $+\mathrm{G}$ argumentation theory in the context of multimorbidity, lies in identifying how and where treatment conflicts arise in the clinical process leading up to a goal. The attacks in Fig. 1 are mostly rebuttals appearing from the drug- 
drug interactions caused by the group sulf, meg, and met and the group acei and $c c b$ and the contrary effects of treatments. From Fig. 1, it is also possible to identify that the argument for met $\left(\mathrm{D}_{1}\right)$ is also rebutted by an argument $\left(\mathrm{E}_{4}\right)$ resulting from the patient having chronic kidney disease and having to delay its progression. This attack is caused by a drug-disease interaction. The only undercutting attacks are made to argument $C_{2}$ by argument $F_{1}^{\prime \prime}$ and to argument $D_{2}$ by argument $\mathrm{F}_{1}^{\prime \prime \prime}$. The arguments attack the applicability of rules $r_{1}$ (used in $\mathrm{C}_{2}$ ) and $r_{2}$ (used in $\mathrm{D}_{2}$ ) in the presence of $c c b$. This type of situation is useful for a physician to know in what circumstances a piece of knowledge is not valid. Going back to attacks brought about by the drug interactions, the attacks highlighted in blue do not result in defeat for $\mathrm{D}_{2}^{\prime \prime} \prec \mathrm{B}_{1}$ and $\mathrm{D} 2^{\prime} \prec \mathrm{C}_{1}$. This happens due to the preferences of the patient expressed in $\leqslant_{\mathcal{R}_{d}}$ which, in turn, are responsible for DefRules $\left(\mathrm{D}_{2}^{\prime \prime}\right) \unlhd_{\mathcal{R}_{d}} \operatorname{DefRules}\left(\mathrm{B}_{1}\right)$ and DefRules $\left(\mathrm{D}_{2}^{\prime}\right) \unlhd_{\mathcal{R}_{d}} \operatorname{DefRules}\left(\mathrm{C}_{1}\right)$, i.e, the use of sulf or meg is preferred to met and arguments that use the latter cannot defeat arguments that use one of the first two. The graph also determines which arguments fulfil the treatment goals established for the patient. By Definitions 6 and 7) we calculate the preferred extensions and respective goal sets:

- $S_{1}=\left\{\mathrm{A}_{1}, \mathrm{~A}_{2}, \mathrm{~A}_{2}^{\prime}, \mathrm{A}_{2}^{\prime \prime \prime}, \mathrm{E}_{1}, \mathrm{E}_{1}^{\prime}, \mathrm{E}_{2}, \mathrm{E}_{3}, \mathrm{E}_{4}, \mathrm{E}_{5}, \mathrm{E}_{6}\right\}$, Goal $\left(S_{1}\right)=\left\{\mathrm{G}_{1}, \mathrm{G}_{3}, \mathrm{G}_{4}\right\}$;

$-S_{2} \quad=\quad\left\{\mathrm{A}_{1}, \mathrm{~A}_{2}, \mathrm{~A}_{2}^{\prime}, \mathrm{A}_{2}^{\prime \prime \prime}, \mathrm{D}_{1}, \mathrm{D}_{2}^{\prime}, \mathrm{D}_{2}^{\prime \prime}, \mathrm{D} 2^{\prime \prime \prime}, \mathrm{D}_{3}, E_{1}^{\prime}, F_{1}, \mathrm{~F}_{1}^{\prime}, \mathrm{F}_{1}^{\prime \prime}\right.$, $\left.\mathrm{F}^{\prime \prime \prime}, \mathrm{F}_{2}\right\}, \operatorname{Goal}\left(S_{2}\right)=\left\{\mathrm{G}_{1}, \mathrm{G}_{4}\right\}$

$-S_{3}=\left\{\mathrm{A}_{1}, \mathrm{~B}_{1}, \mathrm{~B}_{2}, \mathrm{~B}_{2}^{\prime}, \mathrm{B}_{2}^{\prime \prime \prime}, \mathrm{B}_{2}^{\prime \prime \prime \prime}, \mathrm{E}_{1}, \mathrm{E}_{1}^{\prime}, \mathrm{E}_{2}, \mathrm{E}_{3}, \mathrm{E}_{4}, \mathrm{E}_{5}, \mathrm{E}_{6}\right\}, \operatorname{Goal}\left(S_{3}\right)=\left\{\mathrm{G}_{2}, \mathrm{G}_{3}, \mathrm{G}_{4}\right\} ;$

$-S_{4}=\left\{\mathrm{A}_{1}, \mathrm{~B}_{1}, \mathrm{~B}_{2}, \mathrm{~B}_{2}^{\prime}, \mathrm{B}_{2}^{\prime \prime \prime}, \mathrm{B}_{2}^{\prime \prime \prime \prime}, \mathrm{E}_{1}^{\prime}, \mathrm{F}_{1}, \mathrm{~F}_{1}^{\prime}, \mathrm{F}_{1}^{\prime \prime}, \mathrm{F}_{1}^{\prime \prime \prime}, \mathrm{F}_{2}\right\}, \operatorname{Goal}\left(S_{4}\right)=\left\{\mathrm{G}_{2}, \mathrm{G}_{4}\right\}$;

$-S_{5} \quad=\quad\left\{\mathrm{A}_{1}, \mathrm{C}_{1}, \mathrm{C}_{2}, \mathrm{C}_{2}^{\prime}, \mathrm{C}_{2}^{\prime \prime \prime}, \mathrm{C}_{2}^{\prime \prime \prime \prime}, \mathrm{E}_{1}, \mathrm{E}_{1}^{\prime}, \mathrm{E}_{2}, \mathrm{E}_{2}, \mathrm{E}_{3}, \mathrm{E}_{4}, \mathrm{E}_{5}\right.$, $\left.\mathrm{E}_{6}\right\}, \operatorname{Goal}\left(S_{5}\right)=\left\{\mathrm{G}_{2}, \mathrm{G}_{3}, \mathrm{G}_{4}\right\}$;

- $S_{6}=\left\{\mathrm{A}_{1}, \mathrm{C}_{1}, \mathrm{C}_{2}^{\prime}, \mathrm{C}_{2}^{\prime \prime \prime}, \mathrm{C}_{2}^{\prime \prime \prime \prime}, \mathrm{E}_{1}^{\prime}, \mathrm{F}_{1}, \mathrm{~F}_{1}^{\prime}, \mathrm{F}_{1}^{\prime \prime}, \mathrm{F}_{1}^{\prime \prime \prime}, \mathrm{F}_{2}\right\}$, Goal $\left(S_{6}\right)=\left\{\mathrm{G}_{4}\right\}$.

There are six possible solutions for the argumentation theory in the form of preferred extensions: $S_{1}-S_{6}$. Considering the already established goal ordering of $\leqslant_{\mathcal{G}}$, by Definition 77, we calculate the goal set ordering $\unlhd_{G}$. Since extension $S_{1}$ fulfils $w d, b p d$ and $d c k d$, by Definition 8 it is the top preferred extension. This is the case due to the respective goal extension fulfilling the most preferred combination of goals and being the largest doing so. This means that in Example 1, patient A should practice diet and exercise and take angiotensin converting enzyme inhibitor to address obesity, hypertension and delay the progression of kidney disease. In this way, the ASPIC $+\mathrm{G}$ argumentation system ensures that the most important goals in the treatment process are achieved.

\subsection{Explanation of Results}

The explainable nature of argumentation, as analyzed in 828 , also contributes to making it a useful tool in the domain. We do not intend to exhaustively show explanatory properties of ASPIC $+\mathrm{G}$ and leave this aspect for future work. Nonetheless, we present a feature that puts it in advantage in the later comparison with MCDM. We show how one can justify a given formula concluded by a top preferred extension using the notions of defense and sub-argument. In the upcoming formalization we will resort to a defends relation with the following definition. 


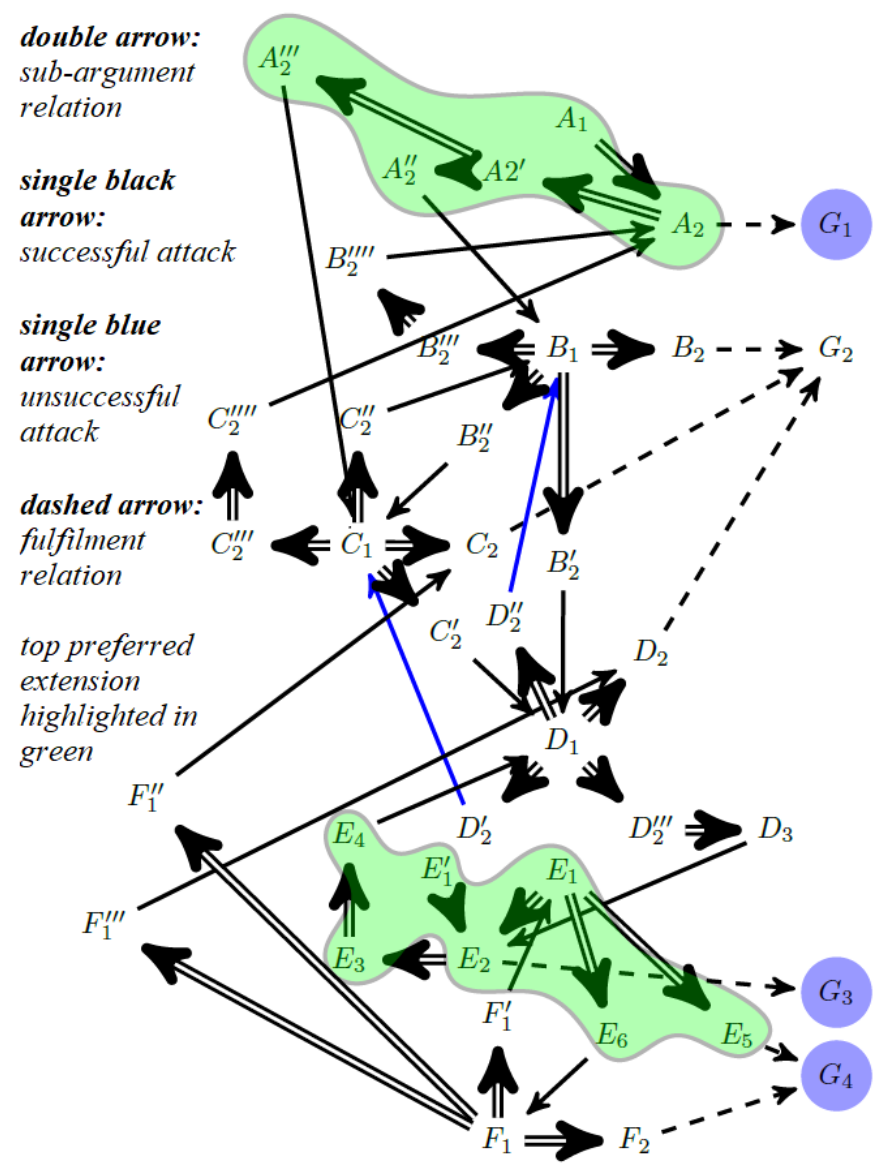

Fig. 1. Argumentation graph for Example 1. 
Definition 10. Let $F=\left(\mathcal{A}, \mathcal{D}, \mathcal{G}, \leqslant_{\mathcal{G}}, \mathcal{F}\right)$ be an $A S P I C+G$ argumentation framework. An argument $\mathrm{C} \in \mathcal{A}$ defends an argument $\mathrm{A} \in \mathcal{A}$ iff: there exists an argument $\mathrm{B} \in \mathcal{A}$ s.t. $\mathrm{B}$ attacks $\mathrm{A}$ and $\mathrm{C}$ attacks $\mathrm{B}$; or there exists an argument $\mathrm{B} \in A$ s.t. $\mathrm{B}$ defends $\mathrm{A}$ and $\mathrm{C}$ defends $\mathrm{B}$.

As mentioned, we regard an explanation for a conclusion as a justification and define it as follows.

Definition 11. Let $F=\left(\mathcal{A}, \mathcal{D}, \mathcal{G}, \leqslant_{\mathcal{G}}, \mathcal{F}\right)$ be an $A S P I C+G$ argumentation framework generated from some argumentation theory and $S$ a top preferred extension of $F$. The possible explanations of a conclusion $\phi$, with $\operatorname{Conc}(\mathrm{A})=\phi$ for some $\mathrm{A} \in S$, is the set $\operatorname{Exp}(\phi, S)=\{\operatorname{Sub}(\mathrm{A}) \cup\{b \in \operatorname{Sub}(\mathrm{B}) \mid \mathrm{B}$ defends $\mathrm{A}\} \mid \mathrm{A} \in S$ s.t. $\operatorname{Conc}(\mathrm{A})=\phi\}$.

Thus, an explanation $\operatorname{Exp}(\phi, S)$ contains all the support (in the form of subarguments) and defense for a conclusion.

Example 2. Let $F=\left(\mathcal{A}, \mathcal{D}, \mathcal{G}, \leqslant_{\mathcal{G}}, \mathcal{F}\right)$ be the ASPIC $+\mathrm{G}$ argumentation framework for Example 1 and $S_{1}$ its top preferred extension. The only explanation for top goal $\left(\mathrm{G}_{1}: w d\right)=\operatorname{Conc}\left(\mathrm{A}_{2}\right)$ in $\operatorname{Exp}\left(w d, S_{1}\right)$ is the set $\left\{\mathrm{A}_{1}, \mathrm{~A}_{2}, \mathrm{~A}_{2}^{\prime}, \mathrm{A}_{2}^{\prime \prime}, \mathrm{A}_{2}^{\prime \prime \prime}\right\}$. Transforming this set into one containing its conclusions yields $\{d e, w d, \neg w i$, $\neg$ sulf,$\neg m e g\}$.

In the explanation of Example 2, $\mathrm{A}_{1}$ is a sub-argument of $\mathrm{A}_{2}$ an therefore supports it. $A_{2}^{\prime}$ defends $A_{2}$ by being a sub-argument of both $A_{2}^{\prime \prime}$ and $A_{2}^{\prime \prime \prime}$, which, in turn, defend $A_{2}$ by attacking $B_{1}$ and $C_{1}$ respectively. From the conclusions, de supports $w d$, and by concluding $\neg w i$, we are also concluding $\neg$ sulf and $\neg m e g$, which are drugs that cause weight increase. Therefore, these arguments justify the fulfilment of weight decrease. With Definition 11 it becomes possible to explain why goals are fulfilled and why treatments are in the solution for patient management. Note that generating compound explanations for any combination of conclusions amounts to performing the union of their respective single explanations.

Some interesting properties of explanations include the closure under the sub-argument relation and direct consistency. The first ensures that for every argument in an explanation, all of its sub-arguments are also included in the explanation, and one can see that it is satisfied by the way the explanation sets are constructed. This property, together with the transitive nature of the notion of defense, ensures that every explanation also contains an explanation for every single one of its sub-conclusions, and hence provides maximal depth for the explanation. On the other hand, direct consistency guaranties that no two arguments in an explanation have opposite conclusions, and follows from the consistency of preferred extensions in ASPIC+ 13 , since explanations are subsets of preferred extensions. Note that this last remark also implies that explanations are indirectly consistent, i.e. even after applying as many strict rules as desired to form new arguments from the ones present in an explanation, it is impossible to find two arguments with opposite conclusions. This ensures the well-behavior of the explanatory feature. 


\section{Relation with Multiple Criteria Decision}

Our argumentation system can also be used to solve MCDM problems. There are numerous variations of MCDM methods [23, but there is no clear method proposed for health care, only a set of guidelines on how to conduct such an analysis, mainly criteria elicitation [12, which are not within the scope of this paper. Therefore, in this comparison we will focus on a general MCDM problem, defined as in [14.

Definition 12. A multiple-criteria decision problem $P=(D, C, a g g)$ consists of:

1. A sequence of decisions $D=\left(d_{1}, \ldots, d_{n}\right)$;

2. A sequence of criteria $C=\left(c_{1}, \ldots, c_{k}\right)$, where each $c_{i} \in C$ is a function $c_{i}$ : $D \rightarrow \mathbb{R}$

3. An aggregation function agg: $\mathbb{R}^{|D| \times|C|} \rightarrow \mathbb{R}^{|D|}$.

We denote with $V_{P}$ the two-dimensional vector of the criteria values for each decision:

$$
V_{P}=\left[\begin{array}{ccc}
c_{1}\left(d_{1}\right) & \ldots & c_{k}\left(d_{1}\right) \\
\vdots & \ddots & \\
c_{1}\left(d_{n}\right) & & c_{k}\left(d_{n}\right)
\end{array}\right]
$$

In MCDM, a decision which is at least as good as every other one according to the aggregation function agg is called a preferred decision.

Definition 13. Given a multiple-criteria decision problem $P=(D, C$, agg $)$, a decision $d_{i} \in D$ is preferred iff for all $d_{j} \in D$ agg $\left(V_{P}\right)_{j} \leq \operatorname{agg}\left(V_{P}\right)_{i}$.

We now provide a mapping to translate a problem into an argumentation theory in ASPIC+G, with a similar construction to the one done in [14.

Definition 14. Let $P=(D, C, a g g)$ be a multiple-criteria decision problem. We construct the argumentation theory $P^{\prime}=\left(\mathcal{A}, \mathcal{D}, \mathcal{G}, \leqslant_{\mathcal{G}}, \mathcal{F}\right)$, such that:

1. $\mathcal{L}$ is the smallest set closed under negation which contains all elements of $D$ and $\mathbb{R}$;

2. $\mathcal{R}=\mathcal{R}_{1} \cup \mathcal{R}_{2} \cup \mathcal{R}_{3} \cup \mathcal{R}_{4}$, where: a) $\mathcal{R}_{1}=\left\{\Rightarrow d_{i} \mid d_{i} \in D\right\}$; b) $\mathcal{R}_{2}=$ $\left\{d_{i} \rightarrow \neg d_{j} \mid d_{i}, d_{j} \in D\right\} ;$ c) $\left.\mathcal{R}_{3}=\left\{d_{i} \rightarrow v_{i, j} \mid d_{i} \in D, v_{i, j} \in V_{P}\right\} ; d\right)$ $\mathcal{R}_{4}=\left\{v_{i, 1}, \ldots, v_{i, k} \rightarrow \operatorname{agg}\left(V_{P}\right)_{i}\left|v_{i, j} \in V_{P}, k=\right| C \mid\right\}$.

3. $\mathrm{n}$ is the empty function;

4. $\leq_{\mathcal{R}_{d}}=\emptyset$;

5. $\mathcal{G}=\left\{\operatorname{agg}\left(V_{P}\right)_{i} \mid d_{i} \in D\right\}$;

6. $\operatorname{agg}\left(V_{P}\right)_{i} \leq_{\mathcal{G}} \operatorname{agg}\left(V_{P}\right)_{j}$ iff $\operatorname{agg}\left(V_{P}\right)_{i} \leq \operatorname{agg}\left(V_{P}\right)_{j}$.

In the resulting argumentation theory $P^{\prime}$, each decision $d_{i}$ gives rise to a series of arguments which eventually lead to the fulfilment of the respective goal $\operatorname{agg}\left(V_{P}\right)_{i}$. The preferred decisions are then retrieved in ASPIC $+\mathrm{G}$ in the form of top preferred extensions thanks to the ordering on the goals. 
Theorem 1. Let $P=(D, C$, agg $)$ be a multiple-criteria decision problem and $P^{\prime}$ its mapping into an argumentation theory in $A S P I C+G$ as defined in Def. 14. Then, for all $d \in D, d$ is a preferred decision in $P$ iff there exists a top preferred extension in $P^{\prime}$ containing the argument $\Rightarrow d$.

The proof of this theorem lies in the fact that all decisions are in conflict with each other thanks to the rules in $\mathcal{R}_{2}$. These being the only conflicts present in the framework, together with the lack of preferences over defeasible rules, ensures that every preferred extension represents exactly one decision and its consequences. By using the ranking over goals in ASPIC $+\mathrm{G}$, which is derived from the ranking over the aggregations in $P$, we filter out the preferred extensions which do not represent preferred decisions, and hence obtain a bijection between preferred decisions in $P$ and top preferred extensions in $P^{\prime} .8$ This shows that ASPIC+G subsumes MCDM and provides thus at least as much expressive power. Additionally, our argumentative approach provides more transparency in the reasoning process and allows for explanations of preferred decisions (top preferred extensions), which is of extreme importance in medical reasoning for the sake of clarity and compliance with recommendations. Within a multimorbidity context, an MCDM method would produce a decision consisting of a set containing recommended treatments and respective aggregated score, which cannot be further decomposed and analyzed to demonstrate how that decision is brought about.

\section{Related Work}

Wilk et al. 26 propose a first order-logic framework in order to detect and mitigate adverse interactions (both drug-drug and drug-disease) between concurrently applied recommendations based on constraint logic programming. Reasoning requires that all stable solutions be encoded beforehand in the form of revision operators and computation mechanism is undecidable, as opposed to the computation of preferred extensions. Zamborlini et al. [27] use their transitionbased medical recommendation model to represent interactions in association with recommendations from different CIGs. Interaction types are defined extensively. However, there is no reasoning mechanism to deal with conflicting recommendations. Spiotta et al. 21] propose a framework to analyze the temporal conformance of followed actions against a single CIG. Using answer set programming they provide explanations on conflicting situations, based on events in the state of a patient. There, the setting is different from the presented herein and the method does not aim to combine different CIGs.

Regarding argumentative approaches, Fox et al. 9] introduce argumentation to help physicians decide for or against treatments. There, patient preferences and patient-specific goals are not featured. Hunter and Williams [11] offer a formal approach to aggregating clinical evidence. Based on the available evidence, arguments are generated for claiming that one treatment is superior, or

\footnotetext{
8 The complete proof is provided as supplemental material.
} 
equivalent, to another. This approach does not concern multimorbidity nor the combination of different CIG recommendations. Brando et al. [5] developed an argumentation-based decision support system which can be used to both represent medical decisions within a single guideline and dynamically choose the most suitable plans to achieve a unique goal. Goal prioritization is not featured.

Amgoud and Prade [1] propose an abstract argument-based framework for decision-making. They formulate a series of decision principles that are goaldriven. Yet, this argumentation framework would not fit decision-making in multimorbidity particularly well for it does not provide sub-argument structure, contrasting to our interest in showing a mapping from CIGs to rule patterns in argumentation. The notions of pro and con arguments are used to evaluate options, while our mechanism is solely based on explicit goal preferences and does not require the user to elicit all possible solutions beforehand. Muller and Hunter [14] formulate a structured argumentation framework for decision-making where goals are used to select decisions. In their work, there is a form of backward reasoning from goals to arguments and a direct comparison of their framework with MCDM. Given the importance of the latter in medical reasoning, we adapt their procedure to perform our own comparison. Note that the type of reasoning performed in our work goes in the opposite direction, from arguments to goals. We first check what are the possible preferred extensions, which, within the context, provide all possible treatment solutions without conflict and respective effects, and then verify which goals each solution fulfils in order to determine its ranking. This kind of reasoning and prioritization of goals are more adequate for assessing the recommendations proposed by CIG agents in a multimorbidity, as these recommendations are already the product of a reasoning process within the CIG agent. Black and Atkinson [4] present a dialogical argumentation framework for reasoning among different agents. Each agent, according to its perspective, has an input as to how a goal can be achieved. ASPIC + G does not possess this dialogical nature, nor it it an objective in the current presentation. Furthermore, Black and Atkinson 4 do not specify an argument evaluation method, whereas our approach establishes detailed semantics for ordering preferred extensions based on respective goal sets.

\section{Conclusions and Future Work}

ASPIC+G models discussions driven by goals, where it is not only important to have explanatory arguments in favour or against a position, but also to know where paths lead to. As such, the presented argumentation system is a contribution in medical reasoning as it is fit for reasoning in multimorbidity. It combines the recommendations of agents, deriving drug-drug and drug-disease conflicts that arise from them, using patient preferences over treatments and preferred semantics so resolve the conflicts and produce solutions, then selecting the best solution based on patient-specific goal preferences. We also show that best solutions are capable of providing explanations in the form of sub-argument and defending argument sets, which are closed under the sub-argument relation, and 
both directly and indirectly consistent. ASPIC+G subsumes MCDM in producing preferred solutions, with the advantage of being more explanatory, an important feature in CDSSs. The present work does not specify preference elicitation or goal prioritization methods. We plan to address this and manage different types of preferences, mainly over defeasible rules, stemming from different sources. An example to be considered is the strength of evidence backing recommendations in relation to effects.

\section{References}

1. Leila Amgoud and Henri Prade. Using arguments for making and explaining decisions. Artificial Intelligence, 173(3-4):413-436, 2009.

2. Katie Atkinson, Trevor Bench-Capon, and Sanjay Modgil. Argumentation for decision support. In International Conference on Database and Expert Systems Applications, pages 822-831. Springer, 2006.

3. Rob Baltussen and Louis Niessen. Priority setting of health interventions: The need for multi-criteria decision analysis. Cost Effectiveness and Resource Allocation, $4: 1-9,2006$.

4. Elizabeth Black and Katie Atkinson. Dialogues that account for different perspectives in collaborative argumentation. In Proceedings of The 8th International Conference on Autonomous Agents and Multiagent Systems, volume 2, pages 867$874,2009$.

5. Maria Brando, David Glasspool, and Aziz Boxwala. Argumentation logic for the flexible enactment of goal-based medical guidelines. Journal of Biomedical Informatics, 45(5):938-949, 2012.

6. David R. Button and John Fox. The syntax and semantics of the PRO forma guideline modeling language. Journal of the American Medical Informatics Association, 10(5):433-443, 2003.

7. Phan Minh Dung. On the acceptability of arguments and its fundamental role in nonmonotonic reasoning, logic programming and n-person games. Artificial Intelligence, 77(2):321-357, 1995.

8. Xiuyi Fan and Francesca Toni. On computing explanations in argumentation. In AAAI, pages 1496-1502, 2015.

9. John Fox, Liz Black, David Glasspool, Sanjay Modgil, Ayelet Oettinger, Vivek Patkar, and Matthew Williams. Towards a general model for argumentation services. In AAAI Spring Symposium: Argumentation for Consumers of Healthcare, pages 52-57, 2006.

10. Paolo Fraccaro, Mercedes Arguello Casteleiro, John Ainsworth, and Iain Buchan. Adoption of Clinical Decision Support in Multimorbidity: A Systematic Review. JMIR Medical Informatics, 3(1):e4, jan 2015.

11. Anthony Hunter and Matthew Williams. Aggregating evidence about the positive and negative effects of treatments. Artificial Intelligence in Medicine, 56(3):173$190,2012$.

12. Kevin Marsh, Maarten Ijzerman, Praveen Thokala, Rob Baltussen, and et al. Multiple Criteria Decision Analysis for Health Care Decision Making - Emerging Good Practices: Report 2 of the ISPOR MCDA Emerging Good Practices Task Force. Value in Health, 19(2):125-137, 2016.

13. Sanjay Modgil and Henry Prakken. The ASPIC + framework for structured argumentation: A tutorial. Argument and Computation, 5(1):31-62, 2014. 
14. Jann Muller and Anthony Hunter. An argumentation-based approach for decision making. In Proceedings of the 2012 IEEE 24th International Conference on Tools with Artificial Intelligence - Volume 01, ICTAI '12, pages 564-571, Washington, DC, USA, 2012. IEEE Computer Society.

15. Christiane Muth, Marjan van den Akker, Jeanet W. Blom, Christian D. Mallen, Justine Rochon, François G. Schellevis, Annette Becker, Martin Beyer, Jochen Gensichen, Hanna Kirchner, Rafael Perera, Alexandra Prados-Torres, Martin Scherer, Ulrich Thiem, Hendrik van den Bussche, and Paul P. Glasziou. The Ariadne principles: How to handle multimorbidity in primary care consultations. BMC Medicine, 12(1):1-11, 2014.

16. NICE . Chronic kidney disease in adults : assessment and management. Technical Report January, 2015.

17. NICE. Hypertension in adults: diagnosis and Hypertension in adults: diagnosis and management management Y Your responsibility our responsibility. Technical Report August 2011, 2011.

18. NICE. Obesity: identification, assessment and management management Y Your responsibility our responsibility. Technical Report November 2014, 2014.

19. NICE. Type 2 diabetes in adults: management. NICE guideline (NG 28). Technical Report December 2015, 2015.

20. Mor Peleg. Computer-interpretable clinical guidelines: A methodological review. Journal of Biomedical Informatics, 46(4):744-63, aug 2013.

21. M. Spiotta, P. Terenziani, and D. T. Dupré. Temporal conformance analysis and explanation of clinical guidelines execution: An answer set programming approach. IEEE Transactions on Knowledge and Data Engineering, 29(11):2567-2580, Nov 2017.

22. G. Spollett. Case Study: A Patient With Uncontrolled Type 2 Diabetes and Complex Comorbidities Whose Diabetes Care Is Managed by an Advanced Practice Nurse. Diabetes Spectrum, 16(1):32-36, 2003.

23. Evangelos Triantaphyllou. Multi-Criteria Decision Making Methods, pages 5-21. Springer US, Boston, MA, 2000.

24. Neeltje P. C. A. Vermunt, Mirjam Harmsen, Gert P. Westert, Marcel G. M. Olde Rikkert, and Marjan J. Faber. Collaborative goal setting with elderly patients with chronic disease or multimorbidity: a systematic review. BMC Geriatrics, 17(1):167, 2017.

25. Emma Wallace, Chris Salisbury, Bruce Guthrie, Cliona Lewis, Tom Fahey, and Susan M Smith. Managing patients with multimorbidity in primary care. BMJ, $350,2015$.

26. Szymon Wilk, Martin Michalowski, Wojtek Michalowski, Daniela Rosu, Marc Carrier, and Mounira Kezadri-Hamiaz. Comprehensive mitigation framework for concurrent application of multiple clinical practice guidelines. Journal of Biomedical Informatics, 66:52-71, 2017.

27. Veruska Zamborlini, Marcos da Silveira, Cedric Pruski, Annette ten Teije, Edwin Geleijn, Marike van der Leeden, Martijn Stuiver, and Frank van Harmelen. Analyzing interactions on combining multiple clinical guidelines. Artificial Intelligence in Medicine, 81:78-93, 2017.

28. Zhiwei Zeng, Xiuyi Fan, Chunyan Miao, Cyril Leung, Chin Jing Jih, and Ong Yew Soon. Context-based and explainable decision making with argumentation. In Proceedings AAMAS '18, pages 1114-1122, 2018. 